\title{
The central mechanism of acupuncture treatment with a long-lasting effect for functional dyspepsia: study protocol for a randomized controlled trial
}

Ruirui Sun ${ }^{1 \dagger}$, Xiaojuan Hong ${ }^{1 \dagger}$, Jing Guo ${ }^{1 \dagger}$, Shuai Yin ${ }^{2}$, Peiming Feng ${ }^{3}$, Lei Lan ${ }^{1}$, Du Lei ${ }^{4}$, Xiaoyan Liu' ${ }^{1}$ Xueling Suo ${ }^{4}$, Tao Yin', Tingting Zhang ${ }^{1}$, Liuyang Huang ${ }^{1}$, Feifei Gao ${ }^{1}$, Qiyong Gong ${ }^{4^{*}}$, Fanrong Liang ${ }^{1 *}$ and Fang Zeng ${ }^{1 *}$

\begin{abstract}
Background: The mechanism of the long-lasting effect and the relationship between the long-lasting effect and the deai sensation, which is the key of achieving the acupuncture effect, has not been well investigated. This trial focuses on investigating the possible central mechanism of the long-lasting effect influenced by the deai sensation.

Methods: A randomized controlled functional brain imaging trial is currently being conducted in Sichuan, China. In total 105 functional dyspepsia (FD) patients will be allocated into three groups: an acupuncture with degi group, an acupuncture without deai group, and a wait-list group. This trial will include a 2-week baseline period, a 4-week treatment period, and a 4-week follow-up period. During the 4-week treatment, patients in two acupuncture groups will receive 20 sessions of acupuncture treatment with or without degi. The Nepean Dyspepsia Index (NDI) and the short form Leeds Dyspepsia Questionnaire (SF-LDQ) will be used to evaluate the clinical efficacy of acupuncture treatment at baseline, the end of treatment, and the end of the follow-up. Functional magnetic resonance imaging (fMRI) scans will be performed to detect cerebral functional changes in 25 patients in each group at three time points mentioned above. The clinical data and fMRI data will be analyzed, respectively. Correlation analysis will be conducted to investigate the relationship between cerebral functional changes and symptom improvement.

Discussion: The results of this trial will allow us to compare the changes of acupuncture therapeutic effect at three time points (the baseline vs. the end of treatment vs. the end of follow-up), and investigate the potential central mechanism of the long-lasting effect influenced by acupuncture with deqi. This trial aims to re-identify the long-lasting effect of acupuncture and investigate its central mechanism, and to further explore the central influence of deai sensation on the long-lasting effect.
\end{abstract}

Trial registration: Chinese Clinical Trial Registry, IDF: ChiCTR-IOR-15006523. Registered on 5 June 2015.

Keywords: Acupuncture, Long-lasting effect, Deqi, Functional dyspepsia, Functional magnetic resonance imaging, Central mechanism, Clinical trial, Protocol

\footnotetext{
* Correspondence: qiyonggong@hmrrc.org.cn; acuresearch@126.com; zeng_fang@126.com

${ }^{\dagger}$ Ruirui Sun, Xiaojuan Hong, and Jing Guo contributed equally to this work.

${ }^{4}$ Huaxi MR Research Center (HMRRC), Departments of Radiology, West China Hospital of Sichuan University, 37\# Guo Xue Xiang, Chengdu 610041, Sichuan, China

${ }^{1}$ Acupuncture and Tuina School/The 3rd Teaching Hospital, Chengdu University of Traditional Chinese Medicine, 37\# Shierqiao Road, Chengdu 610075, Sichuan, China

Full list of author information is available at the end of the article
}

(c) The Author(s). 2018 Open Access This article is distributed under the terms of the Creative Commons Attribution 4.0 International License (http://creativecommons.org/licenses/by/4.0/), which permits unrestricted use, distribution, and reproduction in any medium, provided you give appropriate credit to the original author(s) and the source, provide a link to the Creative Commons license, and indicate if changes were made. The Creative Commons Public Domain Dedication waiver (http://creativecommons.org/publicdomain/zero/1.0/) applies to the data made available in this article, unless otherwise stated. 


\section{Background}

The long-lasting effect of a treatment, also called the "long-term effect," is the perception of a curative effect for a period of time after treatment cessation. It has been widely found following observation after medical therapy [1-3]. For acupuncture treatment, the longlasting effect is considered as one of the most important features in its therapeutic effect, which has been recorded in classical ancient Chinese books and proved by multiple modern clinical trials [4-10]. The long-lasting effect has been reported in trials of acupuncture for treating migraine [10], chronic headache [5], primary insomnia [8], tinnitus [9], etc. Our previous study found that, compared with sham acupuncture, verum acupuncture may be associated with long-term reduction in migraine recurrence [10]. Furthermore, this long-lasting effect is considered to be closely related to the deqi sensation, the key of achieving acupuncture efficacy [11]. In one of our previous randomized clinical trials (RCTs) we found that acupuncture on real acupoints presented persistent effect in the follow-up compared with acupuncture on sham acupoints in patients with functional dyspepsia [4]. However, the central mechanism of the long-lasting effect of acupuncture remains unknown, and its influencing factors have not been well studied.

Functional dyspepsia (FD) is defined as the presence of early satiation, postprandial fullness, epigastric pain, or epigastric burning in the absence of an organic, systemic, or metabolic disease that could explain the symptoms according to the Rome III consensus [12]. It is one of the most common categories in functional gastrointestinal disorders (FGID). In Sweden, the prevalence of FD is between 11 and 20\% [13]. In Asia, it ranges from 8 to 23\% [14]. Although FD is not life-threatening, it significantly influences quality of life (QOL) [15] and leads to high medical costs [16]. Nowadays, many approaches, such as daily proton pump inhibitors (PPI), tricyclic antidepressants, anti-nociceptive agents, and prokinetic agent have been used to treat FD [17-21], but the therapeutic effect is unsatisfactory due to its multi-factor pathology. As a result, more and more patients tends to seek complementary and alternative methods.

We design a randomized controlled functional brain imaging trial with a 10 -week observation period, aiming at: (1) re-identifying the long-lasting effect of acupuncture treatment for FD by comparing the changes of therapeutic effect at three time points (the baseline vs. the end of treatment vs. the end of follow-up); (2) investigating the central mechanism of the long-lasting effect of acupuncture; and (3) exploring the influence of the deqi sensation on the long-lasting effect and its potential central mechanism.

\section{Methods and design \\ Study design}

This is a parallel-group, randomized controlled study. One hundred and five FD patients will be included and randomized with equal allocation to one of the three groups: acupuncture with deqi group, acupuncture without deqi group, and the wait-list group. Among 35 patients in each group, 25 participants will be selected randomly to undergo functional magnetic resonance imaging (fMRI) scans. This trial will include a 2-week baseline period, a 4-week treatment period, and a 4-week follow-up period. During the 4-week treatment, patients in two acupuncture groups will receive 20 sessions of treatments puncturing with or without deqi, while the wait-list group will not receive acupuncture treatment. Both the outcome assessments and fMRI scans will be performed at three time points including: the baseline, the end of the acupuncture treatments, and the end of follow-up (Figs. 1 and 2). This trial is reported in accordance with the Standard Protocol Items: Recommendations for Intervention Trials (SPIRIT) guidelines [22] (Fig. 2; Additional file 1).

\section{Sample size}

According to our previous study, Nepean Dyspepsia Index (NDI) scores of FD patients decreased by 12.2 points after acupuncture and by 7.8 points after sham acupuncture [23]. In this study, we anticipated a reduction of NDI score by 12 points after acupuncture with the deqi sensation and a reduction by 8 points after acupuncture without the deqi sensation, and we anticipated a reduction by 4 points in the wait-list control due to regression to the mean effect. With $\alpha=0.05,1-\beta=0.8$, and a standard deviation of 10 , we needed at least 96 participants in total. Considering a dropout rate of $10 \%$, a total of 105 participants will be included in this trial. According to the sample size of previous bloodoxygenation-level-dependent fMRI (BOLD-fMRI) studies, 20 subjects is the reasonable sample size with stable statistical effect $[24,25]$. Considering the dropout rate and loss of data due to head motion during fMRI scans, 25 participants from each group will receive fMRI scans in this study.

\section{Randomization}

One hundred and five patients will be randomized into three groups by a third party using simple concealed randomization to avoid possible selection bias. With a computer-generated randomization digital table, patients will be informed using sealed envelopes whether they will receive acupuncture treatment and whether they will receive a fMRI scan. The allocation will be concealed to researchers, outcome assessors, and the statistician. 


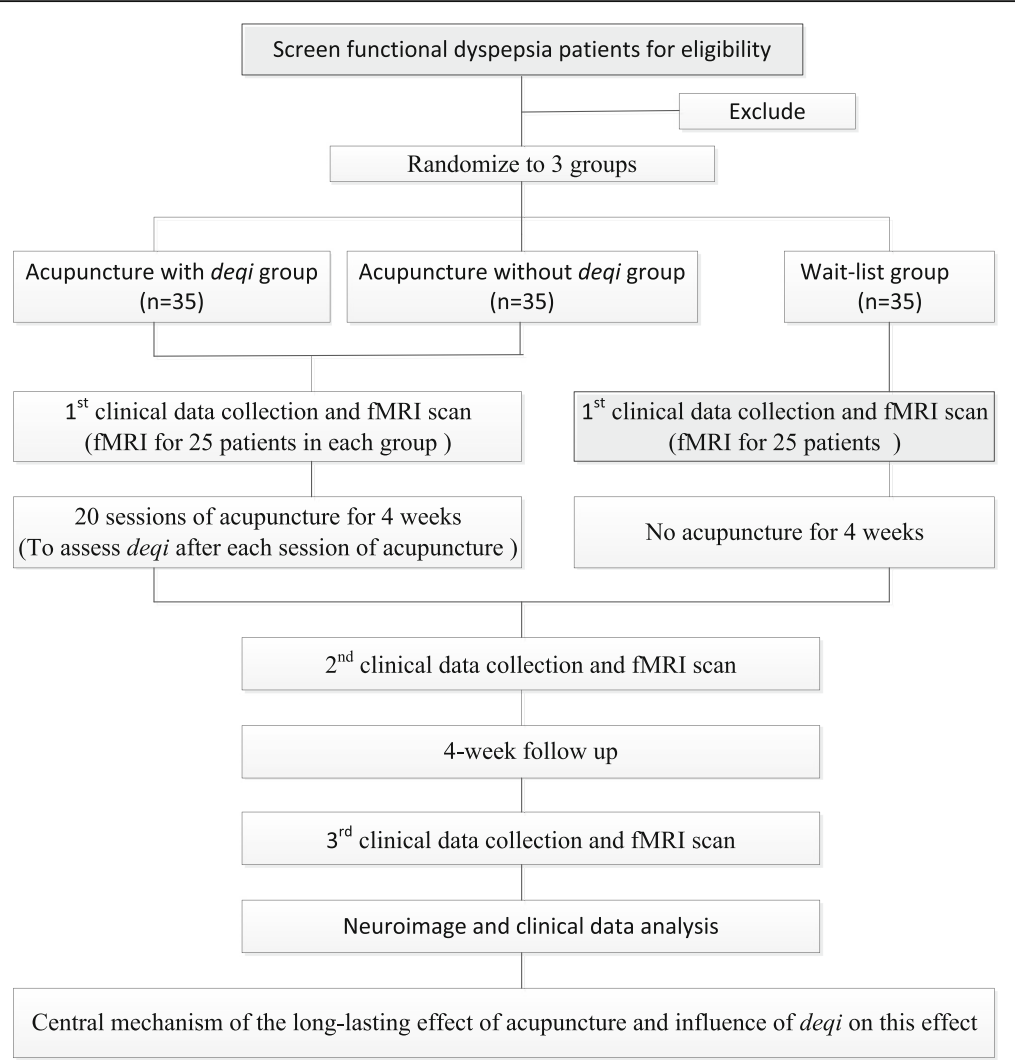

Fig. 1 Study schedule for data collection. The informed consent and examination will be conducted after recruitment. Matched functional dyspepsia (FD) patients will be then randomized into three groups, where two acupuncture groups will receive treatment. Both clinical outcomes and functional magnetic resonance imaging (fMRI) scans will be performed at three time points including: the baseline, the end of acupuncture treatments, and the end of follow-up. Adverse events will be recorded in the case report at any time during the study. a. NDI Nepean Dyspepsia Index, LDQ Leeds Dyspepsia Questionnaire, SAS Self-Rating Anxiety Scale, SDS Self-Rating Depression Scale, BAl Beck Anxiety Inventory, BDI Beck Depression Inventory, VAS Visual Analog Scale

\section{Blinding}

It is difficult to blind patients as well as acupuncturists for the experience of needle manipulation with or without deqi. However, it is feasible to conceal the group assignments from researchers, outcome assessors, and the statistician. Researchers, the acupuncturist, outcome assessors, and the statistician are separate.

\section{Control}

To investigate the influence of the deqi sensation on the long-lasting acupuncture effect, we choose acupuncture without deqi as the control group. In addition, to avoid the influence of a self-healing tendency, we design a wait-list group in which FD patients will not receive acupuncture treatment.

\section{Participants and recruitment}

Based on the Rome III criteria and current academic literature, FD comprises three subtypes: postprandial distress syndrome (PDS), epigastric pain syndrome (EPS), and a subtype with overlapping PDS and EPS features [26]. Among the three subtypes, FD patients diagnosed with PDS are most common in the epidemiology [27], and no significant differences were found in the acupuncture response between FD patients in PDS and FD patients in EPS in our previous study [28]. Therefore, FD patients with PDS, or the subtype with overlapping PDS and EPS features but PDS dominating will be recruited if they match the inclusion criteria. They will be briefly introduced to this study and informed about the benefits as well as possible risks associated with needling (bleeding, hematoma, or fainting). Then, the participants will be required to fill in consent forms and are free to withdraw from the study at any time without any specific reason, penalty or loss of benefits.

All researchers responsible for screening patients will be trained using the Rome III criteria in the pretrial period. They will screen a group of participants and then compare and fix their screening results to achieve homogeneity. 


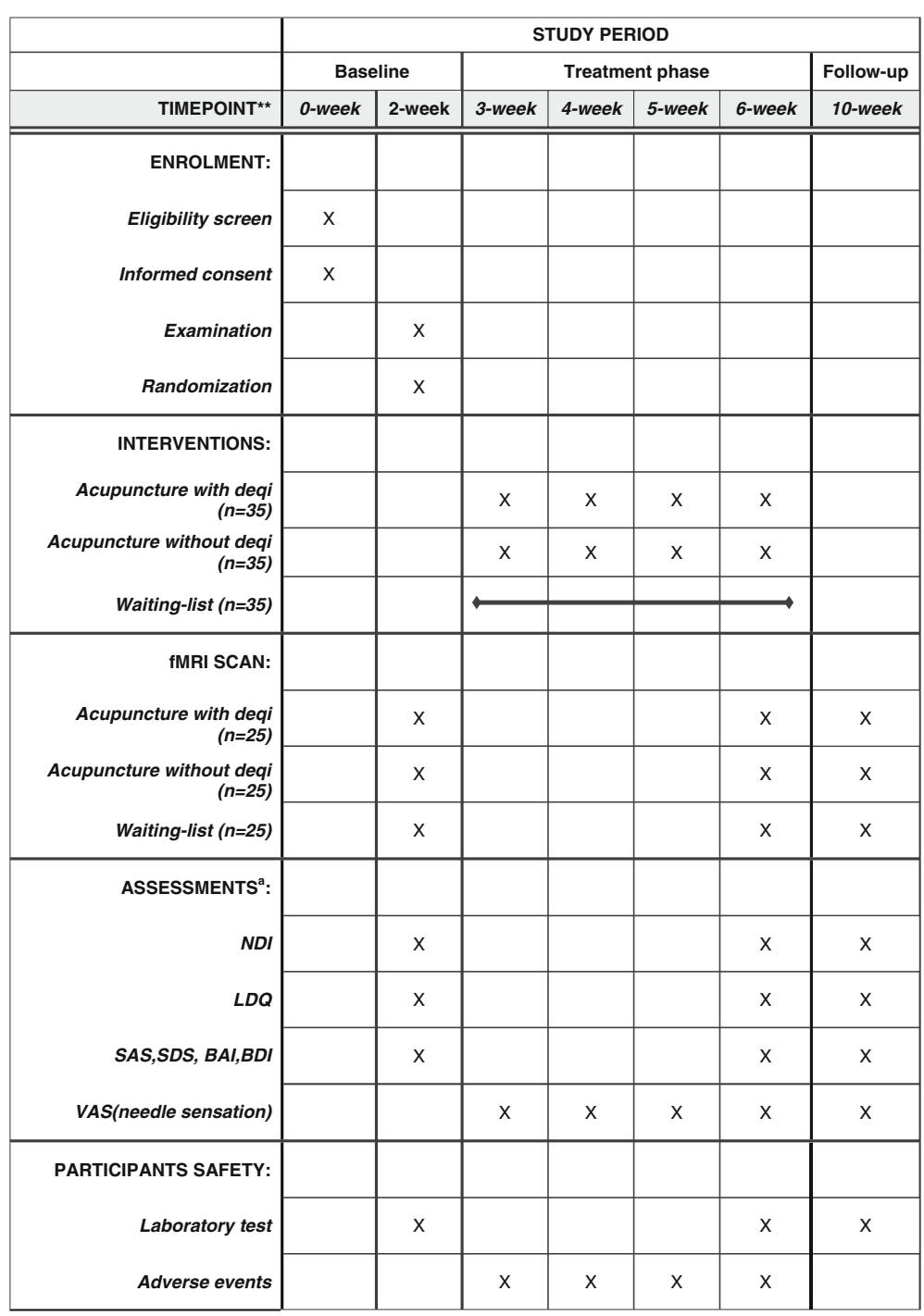

Fig. 2 Standard Protocol Items: Recommendations for Interventional Trials (SPIRIT) schedule of the trial. The present study is a randomized controlled functional brain imaging trial. One hundred and five functional dyspepsia (FD) patients will be included and randomized equally to one of the three groups: the acupuncture with deqi group, the acupuncture without deqi group, and the wait-list group. Among 35 patients in each group, 25 participants will be selected randomly to receive fMRI scans. This trial will include a 2-week baseline period, a 4-week treatment period and a 4-week follow-up period. During the 4-week treatment, patients in two acupuncture groups will receive 20 sessions of treatments, puncturing with or without degi, while the wait-list group will not receive acupuncture. Both the outcome assessments and the fMRI scans will be performed at three time points: baseline, end of acupuncture treatments, and the end of follow-up. The central mechanism of the long-lasting effect and the central role of degi on this effect will be analyzed after data collection

\section{Inclusion criteria}

Participants who meet each of the following criteria will be included: (1) matching the Rome III criteria on FD with subtype PDS or subtype with overlapping PDS and EPS features but PDS dominating; (2) aged between 18 and 45 years, and right handed; (3) without intake of any gastroenteric dynamic drugs in 2 weeks before enrollment; (4) not participating in any other clinical trials; and (5) having signed a written informed consent form.

\section{Exclusion criteria}

Participants who meet one of the following criteria will be excluded: (1) FD with subtype EPS or subtype with overlapping PDS and EPS features but EPS dominating; (2) having esophagitis, gastric atrophy, or erosive gastroduodenal lesions on endoscopy, cholecystitis, gallstones; (3) having psychological problems, being unconscious, having a history of mental disorders, being unable to cooperate in outcome assessment; (4) having aggravating malignant tumors or other serious consumptive diseases, 
infectious, bleeding diseases, etc.; (5) having used aspirin, steroids, nonsteroidal anti-inflammatory drugs, selective serotonin-reuptake inhibitors, medication affecting gastrointestinal motility, or other drugs; (6) pregnant women, or intent to, or in, the breast-feeding period within 6 months; (7) having any contraindications to fMRI scan, such as having a cardiac pacemaker, defibrillator, metal stents, or electronic device in the body, having an intraocular metal foreign body, having claustrophobia, hyperpyrexia, etc.

\section{Recruitment strategies}

All patients will be recruited from outpatients and inpatients of the Digestive Department of the First Teaching Hospital of Chengdu University of Traditional Chinese Medicine (TCM) and the campus of Chengdu University of TCM between January 2016 to December 2018. Patients interested in this trial will be first evaluated by physicians. If they meet the criteria and decide to participate, they will be asked to sign the informed consent. Then, they will be included in this trial for randomization.

\section{Interventions}

The acupoints used in the study include unilateral Zhongwan (CV12) and bilateral Zusanli (ST36) (Fig. 3). The combination of CV12 and ST36 is commonly used for treating FD, and has been used in our previous studies [29].
Acupuncture treatment will be performed by two licensed acupuncturists with clinical experience over 3 years. The acupuncturists will be trained and take an examination before participating in this trial. The two acupuncture groups will receive 20 sessions' treatment over a period of 4 weeks. The acupuncture treatment is performed once per day for 5 days continuously then at 2-day interval.

\section{Acupuncture with the deqi group}

After the skin has been cleaned with tincture iodine and alcohol, the sterile filiform needles $(0.25 \mathrm{~mm}$ in diameter, $40 \mathrm{~mm}$ in length, Hwatuo, Suzhou, China) are inserted perpendicularly into the points for $21-26 \mathrm{~mm}$. Then, the needle is twisted between 90 and $180^{\circ}$, lifted and thrusted in an even amplitude between $0.3 \mathrm{~cm}$ to $0.5 \mathrm{~cm}, 60$ times to 90 times per minute. After the deqi response (including soreness, numbness, distention, and heaviness) is obtained, the needle will be retained in the skin for $30 \mathrm{~min}$, still being manipulated every $10 \mathrm{~min}$ twice for 10 to $15 \mathrm{~s}$.

\section{Acupuncture without the deqi group}

To avoid the deqi response, no needle manipulation will be performed after the perpendicular insertion.

\section{Wait-list group}

Patients will not receive acupuncture treatment during the study. For deqi it is important in achieving clinical

\section{Locations of Acupoints}
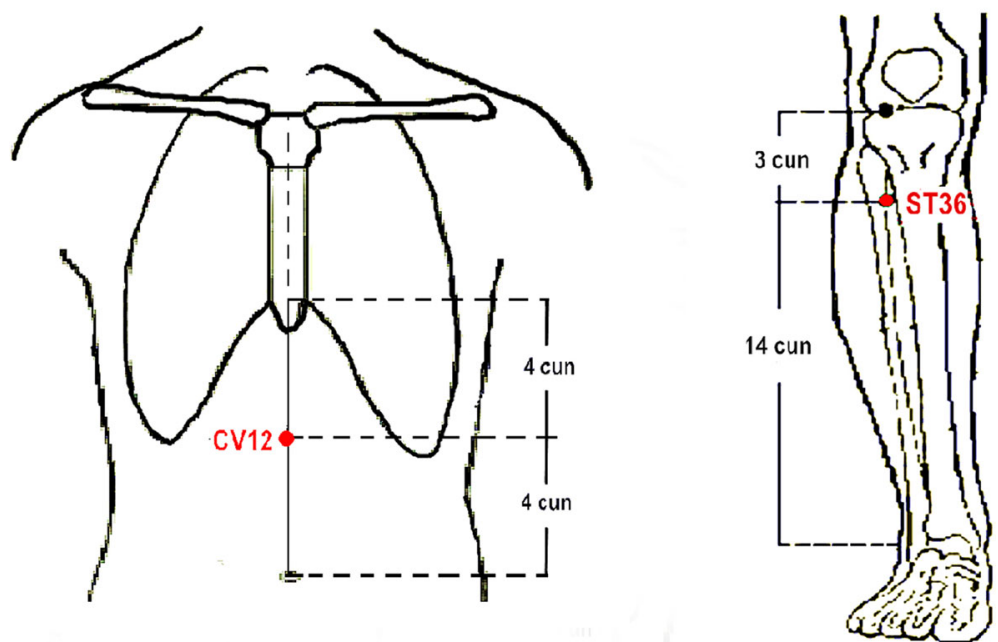

Fig. 3 Locations of acupoints. Locations of acupoints: ST36 (Zusanli), on the anterior lateral side of the shank, 3 cun below ST35 (Dubi), one horizontally placed finger distance lateral to the anterior border of the tibia. CV12 (Zhongwan), on the anterior median line of the upper abdomen, 4 cun above the navel. These two acupoints have been used in our previous study by Shuai Yin et al. (Cerebral mechanism of puncturing at He-Mu point combination for functional dyspepsia: study protocol for a randomized controlled parallel trial. Neural Regen Res. 2017;12: 831-840.), so as to provide a stable acupuncture effect 
efficacy that these patients will be treated by acupuncture with deqi after finishing the study.

Participants with pain, bleeding, fainting, or other severe discomforts should be discontinued from acupuncture treatment and processed immediately. During the acupuncture treatment and follow-up, all participants are usually not allowed to use FD symptomatic or emotional relief medication. But if required, participants can be permitted to receive acute stomach disorder treatment, and the type and dosage of medication used should be recorded in the case report form (CRF).

\section{fMRI scan}

Brain images will be acquired on a 3.0-T magnetic resonance scanner (Siemens, Munich, Germany) at Huaxi Magnetic Resonance Research Center, West China Hospital of Sichuan University, Chengdu, China. The scan will be performed at three time points including: baseline, the end of the treatment, and the follow-up. During the scan, each participant is blindfold, with their ears plugged, and is required to undergo a highresolution, three-dimensional, T1-weighted and BOLDfMRI sequence. Three-dimensional anatomic image parameters are as follows: repetition time/echo time $=$ $1900 \mathrm{~ms} / 2.26 \mathrm{~ms}$, slices $=30$; matrix size $=128 \times 128$, field of view $=256 \times 256 \mathrm{~mm}^{2}$, slice thickness $=1 \mathrm{~mm}$. The BOLD images will be acquired as: repetition time/ echo time $=2000 \mathrm{~ms} / 30 \mathrm{~ms}$, flip angle $=90^{\circ}$, slices $=30$; matrix size $=128 \times 128 \mathrm{~mm}^{2}$, field of view $=240 \times 240$, slice thickness $=5 \mathrm{~mm}$.

\section{Patient safety}

To provide health evaluation before randomization, physical examination for participants will be conducted including gastroscope examination, abdominal ultrasound, blood biochemical test (ALT, AST, BUN, serum creatinine), and electrocardiogram examination. To assess the risks associated with acupuncture, the blood biochemical test will be completed again at the end of treatment. Adverse events, such as pain, bleeding, fainting, or other severe events, should be processed immediately and recorded in the CRF carefully.

\section{Outcome assessments}

The following outcomes will be assessed by independent outcome assessors. These assessors are trained before participating in this trial and blinded to the randomization. Outcome data for participants either completed or discontinued during the study will be collected and recorded.

Clinical outcome assessments include: symptoms, QOL, and psychosocial state. All evaluations will be performed at three time points (the baseline, the end of 20 sessions of acupuncture and the end of follow-up, respectively) (Fig. 2).

The Nepean Dyspepsia Index (NDI) is primarily used to measure the symptoms and QOL for FD patients [30]. It includes the following two parts. The symptom checklist measures 15 upper gastrointestinal symptoms regarding frequency, intensity, and level of discomfort. The QOL checklist includes four aspects, namely life interference (13 items), knowledge/control (seven items), eating/drinking (three items), and sleep/disturbance (two items). Higher scores indicate more severe symptoms and poorer QOL. Our prior research found that the Chinese version of the NDI was reliable and valid for measuring QOL in Chinese FD patients [31].

The short form Leeds Dyspepsia Questionnaire (SF-LDQ) [32] is also performed to assess the symptom severity. The SF-LDQ is a validated and reliable tool with five questions instrument to measure the patients' dyspeptic symptoms. Five questions are used for five symptoms including epigastric pain, postprandial distention, indigestion, heart-burn, and nausea. By using a five-point Likert scale, the symptoms are graded for severity from very mild to very severe: no symptoms $(0$ point), very mild symptoms without influence of regular work (1 point), mild symptoms with influence of regular work ( 2 points), moderate symptoms (3 points), severe symptoms (4 points), and extremely severe symptoms (5 points). The severity of dyspepsia is evaluated by total scores on these five symptoms. Higher total scores indicate more severe symptoms.

As emotional changes, such as anxiety and depression, are commonly seen accompanying symptoms in FD patient, the Zung Self-Rating Anxiety Scale (SAS) [33], the Zung Self-Rating Depression Scale (SDS) [34], the Beck Anxiety Inventory (BAI) [35], and the Beck Depression Inventory (BDI) [36] are used in this study to evaluate the psychological condition of patients.

\section{Needle sensation evaluations}

Participants in the two acupuncture groups will be asked to fill the needle sensation evaluation form after receiving each acupuncture treatment. The form is based on the Chinese version of modified Massachusetts General Hospital Acupuncture Sensation Scale (C-MASS) $[37,38]$, and its validity and reliability have been examined [38, 39]. In the form, the Visual Analog Scale (VAS) [40] is selected to quantitatively evaluate the deqi sensation after each acupuncture treatment. This is a 10-point scale with anchor words "none" (no score), "mild" (about two scores), "moderate" (around five scores), "severe" (almost eight scores), and "unbearable" (nearly ten scores) spaced along the continuum. The scale will be used to access evaluate the deqi sensations (including soreness, numbness, fullness, heaviness, 
aching), respectively. Moreover, if the scale does not completely describe the needle sensations that subjects experienced, subjects can add what they feel in a blank row below the scale.

Since some participants were sensitive while some were not, both acupuncture groups' participants will be re-divided into the "actual deqi group" and "actual without deqi group" according to the median of the mean needle sensation score across 20 times treatment. Then the "actual deqi group" and "actual without deqi group" will be compared with both clinical data and fMRI data.

\section{Data management and monitoring}

The CRFs for each participant will be prepared in both paper and electronic versions. Only outcome assessors have access to CRFs and will perform the double-data entry. The Evidence-based Medicine Center of the university will be responsible for monitoring the study as well as the data every 3 months.

\section{Data analysis}

\section{Clinical data analysis}

The intention-to-treat analysis will be used for all allocated participants in the baseline. This study focuses on the lasting-effect of acupuncture, and the observed data will be used as the primary analysis and last observation-carried-forward (LOCF) analysis will be taken as a sensitivity analysis. Clinical outcome will be analyzed using SPSS 22.0 statistics software (IBM SPSS Statistics, IBM Corp, Somers, NY, USA). Data on Skewed distribution will be normalized. Means, standard deviations, and 95\% confidence intervals (CIs) will be used to describe the continuous data.

The demographic and baseline characteristics among three groups will be compared using one-way analysis of variance (ANOVA). A $\chi^{2}$ test will be used for categorical variables. Due to the occurrence of self-healing tendency in FD, it is important to testify the existence of acupuncture effect. The clinical outcome NDI and SF-LDQ scores will be compared between the acupuncture group and the wait-list group among three time points by using the repeated measures ANOVA (two independent groups with repeated measures). Then, the acupuncture group with and without deqi at three time points will be primarily compared using repeated measures model.

A two-sided test will be used during the analysis, with a significance level of 0.05 . The data will be analyzed by statisticians who are blinded to the test settings.

\section{fMRI data analysis}

The fMRI scan data will be preprocessed and analyzed by SPM12 software (SPM12, Wellcome Department of Imaging Neuroscience, London, UK; http://www.fil.ion.ucl.a c.uk/spm/) performing on MATLAB 8.6 (Mathworks,
Inc., Natick, MA, USA). After data preprocessing, regional homogeneity (ReHo), amplitude of low-frequency fluctuation (ALFF) and functional connectivity (FC) will be used to investigate the different cerebral responses between deqi and without deqi. Then correlation analysis will be conducted between clinical data and fMRI image data.

\section{Discussion}

Based on ancient records and recent RCT results, acupuncture has a long-lasting effect for treating dyspepsia, but its central mechanism and influential factors remain uncertain. Considering the importance of the deqi sensation for the acupuncture effect, we have designed a randomized controlled fMRI trial to explore the central mechanism of the acupuncture long-lasting effect and the influence of the deqi sensation on the long-lasting effect for the first time.

Deqi may play an essential role in achieving the long-lasting effect for its definite influence on acupuncture effect

The long-lasting effect, as one of the most commonly phenomenon and important feature of acupuncture effect, refers to the perception of a curative effect for a period of time after termination of acupuncture treatment. It has been recorded in a number of ancient Chinese medical books and reported in many clinical trials such as acupuncture treatment for migraine [7], FD [4], cervical spondylosis [41], chronic pain in the knee joints [42], and others $[5,6]$. For example, in a systematic review including 13 studies on chronic pain in knee joints, three of them found that the acupuncture efficacy still remained in the following 6 to 12 months after treatment [42]. Our previous multicenter clinical trials on acupuncture treatment for FD also demonstrated that the effects of puncturing on acupoints were not only significantly different from those on non-acupoints at the end of 4 weeks' treatment, but also in the 4 weeks and 12 weeks after the termination of treatment [4]. All the above indicated the existence of the long-lasting effect and its importance in keeping acupuncture efficacy.

Deqi, also called the arrival of $q i$, or the needle sensation, is a series of multidimensional and intense sensations including numbness, soreness, distention, heaviness, and dull pain, etc. that patients experience during acupuncture stimulation [43]. A great amount of ancient acupuncture classics recorded that " the achievement of acupuncture effect depends on the qi arrival" "Huangdi Neijing," The Inner Canon of Huangdi), and "the quicker the $q i$ arrives, the faster the acupuncture effect acquires" ("Biao You Fu," a Poetic Prose on the Elucidation of Acupuncture). Modern clinical trials have considered that deqi plays an essential role in the process of achieving the acupuncture effect [37, 44-47]. For example, a study on Bell's palsy found that acupuncture with deqi can induce a greater 
therapeutic effect, and stronger intensity of deqi was associated with better therapeutic effects [47].

Considering the definite influence of deqi on acupuncture effect, we noted that deqi may play a vital role in achieving the long-lasting effect. Our previous study on migraine also demonstrated that the therapeutic effect induced by acupuncture with deqi can last for 20 weeks after 4 weeks' treatment [48]. The results not only confirmed the existence of the long-lasting effect, but also presented its intimate relationship with deqi. So, this study tries to firstly re-identify the influence of deqi on the long-lasting effect, and then further explore the mechanism of how deqi plays the central role in the long-lasting effect.

\section{The potential central mechanism of the long-lasting effect has not been well investigated}

Although the long-lasting effect has been reported by a number of RCTs [9, 10, 42, 49] and animal studies $[50,51]$, most of them focused on identifying the existence of the long-lasting effect. An animal study on irritable bowel syndrome (IBS) reported that the P2X3 receptor, which played an important role in visceral pain, was downregulated at the sixth week after the electroacupuncture (EA) treatment, suggesting that the regulation of the $\mathrm{P} 2 \mathrm{X} 3$ receptor may be associated with the mechanism underlying the production of the long-lasting effect [51].

Since central integration is the key for achieving acupuncture effect, using functional brain imaging techniques including fMRI, positron emission tomography (PET) / computed tomography $(\mathrm{CT})$, electroencephalography (EEG), and magnetoencephalography (MEG) to explore the central mechanism of acupuncture effect has attracted increasing attention worldwide in the last 20 years. In our previous academic literature study, we found that in total 168 papers on acupuncture functional brain imaging were published from 1995 to 2014 [52]. These studies mapped the brain regions involving in acupuncture effect. For example, Hui. and her co-investigators found that acupuncture with deqi can elicit extensive negative BOLD signal changes (deactivation) in the limbic-paralimbic-neocortical network (LPNN) and positive BOLD signal changes in somatosensory regions of the brain [53]. This study provided functional brain imaging evidence of the influence of deqi on cerebral activity.

However, little work has been done in revealing the potential central mechanism of the long-lasting effect and how deqi influences the production of the long-lasting effect, which is worthy of investigation. Therefore, this trial aims to investigate the central mechanism of the acupuncture long-lasting effect and the central influence of deqi based on re-identifying the existence of the long-lasting effect on acupuncture for FD.

\section{The quality control program is the precondition for the result reliability}

To improve the result reliability of this study, we design the quality control program from the following four aspects: (1) patient selection for the influence of age and handedness on cerebral function and structure have long been investigated, this study restricts the subjects to age between 18 to 45 years and being right-handed for baseline homogeneity; (2) sample size to obtain stable statistical power; we include 35 patients in each group for clinical evaluation and 25 patients in each group for the central mechanism study; and (3) fMRI scan; all scans are performed in the morning with the same scanner and operator. Scans on female participants are performed during the same menstrual cycle. Moreover, during the $24 \mathrm{~h}$ before scanning, participants will be asked to maintain their regular lifestyle and avoid overexertion and staying up late. No permission with alcohol, tobacco, tea, and coffee. Before being scanned, the emotional state of each participant will be evaluated via the emotional state assessment scales. To exclude significant emotional changes, during the scan participants will be asked to close their eyes and use a blindfold and to plug their ears with earplugs, stay awake, and not to speak. The scan room keeps the temperature between 18 and $22{ }^{\circ} \mathrm{C}$, with humidity greater than $60 \%$ and noise less than $150 \mathrm{~dB}$; (4) acupuncture manipulation; the acupuncture treatment will be performed by two experienced acupuncturists using a standard operation procedure. The Deqi sensation will be evaluated and recorded with the VAS after each acupuncture treatment.

In conclusion, the long-lasting effect is an important feature in acupuncture efficacy, and deqi may be one vital factor in producing the long-term effect. This study is the first functional brain imaging study which focuses on exploring the central mechanism of the long-lasting effect and the central influence of deqi on this effect with strict quality control. The results may provide a new approach to investigate the mechanism of the long-lasting effect.

\section{Trial status}

This trial was registered in June 2015 (Registration Number: ChiCTR-IOR-15006523, the protocol version number: F2.0). Due to the funding delay, recruitment, preparation, and other reasons, this trial started at the beginning of 2016. Its deadline would correspondingly be postponed to December 2018. The first participant was included on 18 May 2016. To date, 61 participants have been recruited. This trial is still recruiting patients. The recruitment will be completed by approximately 30 November 2018. 


\section{Additional file}

Additional file 1: Standard Protocol Items: Recommendations for Interventional Trials (SPIRIT) 2013 Checklist: recommended items to address in a clinical trial protocol and related documents*. (DOC $127 \mathrm{~kb}$ )

\begin{abstract}
Abbreviations
ALFF: Amplitude of low-frequency fluctuation; BAl: Beck Anxiety Inventory; BDI: Beck Depression Inventory; BOLD-fMRI: Blood oxygenation level-dependent fMRl; EA: Electroacupuncture; EEG: Electroencephalography; FC: Functional connectivity; FD: Functional dyspepsia; FGID: Functional gastrointestinal disorders; fMRI: Functional magnetic resonance imaging; IBS: Irritable bowel syndrome; LDQ: Leeds Dyspepsia Questionnaire; LPNN: Limbicparalimbic-neocortical network; MEG: Magnetoencephalography; NDI: Nepean Dyspepsia Index; PET/CT: Positron emission tomography/computed tomography; PPI: Proton pump inhibitor; QOL: Quality of life; RCT: Randomized clinical trial; ReHo: Regional homogeneity; SAS: Zung Self-Rating Anxiety Scale; SDS: Zung Self-Rating Depression Scale; SPIRIT: Standard Protocol Items: Recommendations for Intervention Trials; TCM: Traditional Chinese Medicine; VAS: Visual Analog Scale
\end{abstract}

\section{Acknowledgments}

We are grateful to Prof. Yong Tang, Acupuncture and Tuina School, Chengdu University of Traditional Chinese Medicine (TCM), for scientific discussions, and Liangsheng Zhang for English language and grammar editing.

\section{Funding}

The trial is financially supported by the National Natural Science Foundation of China (NO. 81473602); the National Natural Science Foundation of Outstanding Youth Fund in China (NO. 81622052); the Sichuan Youth Science and Technology Foundation (NO. 15QNJJ0008), The Special Fund for the Authors Who win the 100 Top Doctoral Dissertations Award of China (NO. 2014084); the Education Ministry's New Century Excellent Talents Supporting Plan in China (NCET-13-0699); the Ten Thousand Talent Program (W02020595).

\section{Authors' contributions}

$\mathrm{RS}, \mathrm{XH}, \mathrm{JG}, \mathrm{QG}, \mathrm{FL}$, and FZ participated in the conception and design of the trial, in plans for the analysis of the data. $R S, X H$, and JG participated in drafting the manuscript. SY, PF, LL, DL, XL, XS, TY, TZ, LH, and FG participated in the data collection, and were in charge of recruitment and treatment of patients. All authors discussed, read, revised the manuscript, and all approved the publication of this protocol.

\section{Ethics approval and consent to participate}

This study protocol has been approved by the Institutional Review Boards and Ethics Committees of the 1st Teaching Hospital of Chengdu University of TCM with the ethical approval identifier 2015KL-002. This trial is registered in the Chinese Clinical Trial Registry, ChiCTR-IOR-15006523. This trial follows the principles of the Declaration of Helsinki (Version Edinburgh 2000). Only patients who signed the informed consent form will be included. In addition, all researchers were trained and signed a pledge to protect the confidentiality of study participants.

\section{Consent for publication}

Not applicable

\section{Competing interests}

The authors declare that they have no competing interests.

\section{Publisher's Note}

Springer Nature remains neutral with regard to jurisdictional claims in published maps and institutional affiliations.

\section{Author details}

${ }^{1}$ Acupuncture and Tuina School/The 3rd Teaching Hospital, Chengdu University of Traditional Chinese Medicine, 37\# Shierqiao Road, Chengdu 610075, Sichuan, China. ${ }^{2}$ First Affiliated Hospital of Henan University of Traditional Chinese Medicine, Zhengzhou, Henan Province, China. ${ }^{3}$ The 1st Teaching Hospital of Chengdu University of Traditional Chinese Medicine,
Chengdu, Sichuan, China. ${ }^{4}$ Huaxi MR Research Center (HMRRC), Departments of Radiology, West China Hospital of Sichuan University, 37\# Guo Xue Xiang, Chengdu 610041, Sichuan, China.

Received: 7 January 2018 Accepted: 13 June 2018

Published online: 13 July 2018

\section{References}

1. Chen L, Liu D, Zou L, Huang J, Chen J, Zou Y, Lai J, Chen J, Li H, Liu G. Efficacy of high intensity laser therapy in treatment of patients with lumbar disc protrusion: a randomized controlled trial. J Back Musculoskelet Rehabil. 2018;(1):191-96.

2. Choi JY, Jung SC, Sykes JM. Clinical outcome and patients' satisfaction study after otoplasty using hybrid techniques in adult patients. J Craniofac Surg. 2017:28:1278-81.

3. Hazime FA, Baptista AF, de Freitas DG, Monteiro RL, Maretto RL, Hasue RH, Joao SMA. Treating low back pain with combined cerebral and peripheral electrical stimulation: a randomized, double-blind, factorial clinical trial. Eur J Pain. 2017;21:1132-43.

4. Ma TT, Yu SY, Li Y, Liang FR, Tian XP, Zheng H, Yan J, Sun GJ, Chang XR, Zhao L, et al. Randomised clinical trial: an assessment of acupuncture on specific meridian or specific acupoint vs. sham acupuncture for treating functional dyspepsia. Aliment Pharmacol Ther. 2012;35:552-61.

5. Vickers AJ, Rees RW, Zollman CE, McCarney R, Smith CM, Ellis N, Fisher P, Van Haselen R. Acupuncture for chronic headache in primary care: large, pragmatic, randomised trial. BMJ. 2004;328:744.

6. Melchart D, Streng A, Hoppe A, Brinkhaus B, Witt C, Wagenpfeil S, Pfaffenrath V, Hammes M, Hummelsberger J, Irnich D, et al. Acupuncture in patients with tension-type headache: randomised controlled trial. BMJ. 2005;331:376-82.

7. Li Y, Zheng H, Witt CM, Roll S, Yu SG, Yan J, Sun GJ, Zhao L, Huang WJ, Chang $X R$, et al. Acupuncture for migraine prophylaxis: a randomized controlled trial. CMAJ. 2012;184:401-10.

8. Yin X, Gou M, Xu J, Dong B, Yin P, Masquelin F, Wu J, Lao L, Xu S. Efficacy and safety of acupuncture treatment on primary insomnia: a randomized controlled trial. Sleep Med. 2017:37:193-200.

9. Jeon SW, Kim KS, Nam HJ. Long-term effect of acupuncture for treatment of tinnitus: a randomized, patient- and assessor-blind, sham-acupuncturecontrolled, pilot trial. J Altern Complement Med. 2012;18:693-9.

10. Zhao L, Chen J, Li Y, Sun X, Chang X, Zheng H, Gong B, Huang Y, Yang M, Wu $X$, et al. The long-term effect of acupuncture for migraine prophylaxis: a randomized clinical trial. JAMA Intern Med. 2017;177:508-15.

11. Zhou W, Benharash P. Significance of "Degi" response in acupuncture treatment: myth or reality. J Acupunct Meridian Stud. 2014;7:186-9.

12. Tack J, Talley NJ. Functional dyspepsia-symptoms, definitions and validity of the Rome III criteria. Nat Rev Gastroenterol Hepatol. 2013;10:134-41.

13. Aro P, Talley NJ, Johansson SE, Agreus L, Ronkainen J. Anxiety is linked to new-onset dyspepsia in the Swedish population: a 10-year follow-up study. Gastroenterology. 2015:148:928-37.

14. Ghoshal UC, Singh R, Chang FY, Hou X, Wong BC, Kachintorn U. Epidemiology of uninvestigated and functional dyspepsia in Asia: facts and fiction. J Neurogastroenterol Motil. 2011;17:235-44.

15. Talley NJ, Locke GR 3rd, Lahr BD, Zinsmeister AR, Tougas G, Ligozio G, Rojavin MA, Tack J. Functional dyspepsia, delayed gastric emptying, and impaired quality of life. Gut. 2006;55:933-9.

16. Miwa H, Kusano M, Arisawa T, Oshima T, Kato M, Joh T, Suzuki H, Tominaga K, Nakada K, Nagahara A, et al. Evidence-based clinical practice guidelines for functional dyspepsia. J Gastroenterol. 2015;50:125-39.

17. Moayyedi P, Delaney BC, Vakil N, Forman D, Talley NJ. The efficacy of proton pump inhibitors in nonulcer dyspepsia: a systematic review and economic analysis. Gastroenterology. 2004;127:1329-37.

18. Soo S, Forman D, Delaney BC, Moayyedi P. A systematic review of psychological therapies for nonulcer dyspepsia. Am J Gastroenterol. 2004;99:1817-22.

19. Hiyama T, Yoshihara M, Matsuo K, Kusunoki H, Kamada T, Ito M, Tanaka S, Nishi N, Chayama K, Haruma K. Meta-analysis of the effects of prokinetic agents in patients with functional dyspepsia. J Gastroenterol Hepatol. 2007; 22:304-10.

20. Talley NJ, Tack J, Ptak T, Gupta R, Giguere M. Itopride in functional dyspepsia: results of two phase III multicentre, randomised, double-blind, placebocontrolled trials. Gut. 2008;57:740-6.

21. Lacy BE, Talley NJ, Locke GR 3rd, Bouras EP, DiBaise JK, El-Serag HB, Abraham BP, Howden CW, Moayyedi P, Prather C. Review article: 
current treatment options and management of functional dyspepsia. Aliment Pharmacol Ther. 2012;36:3-15.

22. Chan AW, Tetzlaff JM, Gotzsche PC, Altman DG, Mann H, Berlin JA, Dickersin $K$, Hrobjartsson A, Schulz KF, Parulekar WR, et al. SPIRIT 2013 explanation and elaboration: guidance for protocols of clinical trials. BMJ. 2013;346:e7586.

23. Zeng F, Qin W, Ma T, Sun J, Tang Y, Yuan K, Li Y, Liu J, Liu X, Song W, et al. Influence of acupuncture treatment on cerebral activity in functional dyspepsia patients and its relationship with efficacy. Am J Gastroenterol. 2012;107:1236-47.

24. Desmond JE, Glover GH. Estimating sample size in functional MRI (fMRI) neuroimaging studies: statistical power analyses. J Neurosci Methods. 2002; 118:115-28.

25. Hayasaka S, Peiffer AM, Hugenschmidt CE, Laurienti PJ. Power and sample size calculation for neuroimaging studies by non-central random field theory Neurolmage. 2007;37:721-30

26. Enck P, Azpiroz F, Boeckxstaens G, Elsenbruch S, Feinle-Bisset C, Holtmann G, Lackner JM, Ronkainen J, Schemann M, Stengel A, et al. Functional dyspepsia. Nat Rev Dis Primers. 2017;3:17081.

27. Santonicola A, Siniscalchi M, Capone P, Gallotta S, Ciacci C, lovino P. Prevalence of functional dyspepsia and its subgroups in patients with eating disorders. World J Gastroenterol. 2012;18:4379-85.

28. Ma T, Zeng F, Li Y, Wang CM, Tian X, Yu S, Zhao L, Wu X, Yang M, Wang D, Liang F. Which subtype of functional dyspepsia patients responses better to acupuncture? A retrospective analysis of a randomized controlled trial. Forsch Komplementmed. 2015;22:94-100.

29. Yin S, Chen Y, Lei D, R-r S, Ma T-t, P-m F, He Z-X, X-I S, Ma P-h, Y-z Q, et al. Cerebral mechanism of puncturing at $\mathrm{He}-\mathrm{Mu}$ point combination for functional dyspepsia: study protocol for a randomized controlled parallel trial. Neural Regen Res. 2017;12:831-40.

30. Talley NJ, Verlinden M, Jones M. Validity of a new quality of life scale for functional dyspepsia: a United States multicenter trial of the Nepean Dyspepsia Index. Am J Gastroenterol. 1999;94:2390-7.

31. Tian XP, Li Y, Liang FR, Sun GJ, Yan J, Chang XR, Ma TT, Yu SY, Yang XG. Translation and validation of the Nepean Dyspepsia Index for functional dyspepsia in China. World J Gastroenterol. 2009;15:3173-7.

32. Fraser A, Delaney BC, Ford AC, Qume M, Moayyedi P. The short-form Leeds Dyspepsia Questionnaire validation study. Aliment Pharmacol Ther. 2007;25: 477-86.

33. Zung WW. A rating instrument for anxiety disorders. Psychosomatics. 1971; 12:371-9.

34. Zung WW. A self-rating depression scale. Arch Gen Psychiatry. 1965;12:63-70

35. Beck AT, Epstein N, Brown G, Steer RA. An inventory for measuring clinical anxiety: psychometric properties. J Consult Clin Psychol. 1988;56:893-7.

36. Beck AT, Ward CH, Mendelson M, Mock J, Erbaugh J. An inventory for measuring depression. Arch Gen Psychiatry. 1961;4:561-71.

37. Kong J, Gollub R, Huang T, Polich G, Napadow V, Hui K, Vangel M, Rosen B, Kaptchuk TJ. Acupuncture de qi, from qualitative history to quantitative measurement. J Altern Complement Med. 2007;13:1059-70.

38. Yu DT, Jones AY, Pang MY. Development and validation of the Chinese version of the Massachusetts General Hospital Acupuncture Sensation Scale: an exploratory and methodological study. Acupunct Med. 2012;30:214-21.

39. Li M, Yuan H, Wang P, Xin S, Hao J, Liu M, Li J, Yu M, Zhang X. Influences of De ai induced by acupuncture on immediate and accumulated analgesic effects in patients with knee osteoarthritis: study protocol for a randomized controlled trial. Trials. 2017;18:251

40. Wewers ME, Lowe NK. A critical review of visual analogue scales in the measurement of clinical phenomena. Res Nurs Health. 1990;13:227-36.

41. Zhu XP, Fu WB, Zhang GC, et al. Systematic review on the long-term effect of acupuncture treating for cervical spondylosis. Chin J Gerontol. 2011;6: 918-21.

42. White A, Foster NE, Cummings M, Barlas P. Acupuncture treatment for chronic knee pain: a systematic review. Rheumatology (Oxford). 2007:46:384-90.

43. Liu CZ, Litscher G, Liang FR, Kong J, Wang LP, Wang L. Deqi sensation in different kinds of acupuncture 2014. Evid Based Complement Alternat Med. 2015;2015:306138.

44. Takeda W, Wessel J. Acupuncture for the treatment of pain of osteoarthritic knees. Arthritis Care Res. 1994;7:118-22.

45. Park H, Park J, Lee H, Lee H. Does Deai (needle sensation) exist? Am J Chin Med. 2002;30:45-50

46. Kong J, Fufa DT, Gerber AJ, Rosman IS, Vangel MG, Gracely RH, Gollub RL. Psychophysical outcomes from a randomized pilot study of manual, electro, and sham acupuncture treatment on experimentally induced thermal pain. J Pain. 2005;6:55-64

47. Xu SB, Huang B, Zhang CY, Du P, Yuan Q, Bi GJ, Zhang GB, Xie MJ, Luo X, Huang GY, Wang W. Effectiveness of strengthened stimulation during acupuncture for the treatment of Bell palsy: a randomized controlled trial. CMAJ. 2013;185:473-9.

48. Chen J. Evaluating the prophylaxis and the long-term effectiveness of meridian-based acupuncture for migraine: a randomized, controlled trial. Doctoral Dissertation of China 2014 May.

49. Raissi GR, Forogh B, Ahadi T, Ghahramanpoori S, Ghaboussi P, Sajadi S. Evaluation of acupuncture in the treatment of restless legs syndrome: a randomized controlled trial. J Acupunct Meridian Stud. 2017;10:346-50.

50. Zhang R, Andersen AH, Hardy PA, Forman E, Evans A, Ai Y, Yue J, Yue G, Gash DM, Grondin R, Zhang Z. Objectively measuring effects of electroacupuncture in parkinsonian rhesus monkeys. Brain Res. 2018;1678:12-9.

51. Weng ZJ, Wu LY, Zhou CL, Dou CZ, Shi Y, Liu HR, Wu HG. Effect of electroacupuncture on $\mathrm{P} 2 \mathrm{X} 3$ receptor regulation in the peripheral and central nervous systems of rats with visceral pain caused by irritable bowel syndrome. Purinergic Signal. 2015;11:321-9.

52. Qiu $K$, Jing $M$, Sun $R$, Yang J, Liu X, He Z, Yin S, Lan Y, Cheng S, Gao F, et al. The status of the quality control in acupuncture-neuroimaging studies. Evid Based Complement Alternat Med. 2016;2016:3685785.

53. Hui KK, Marina O, Claunch JD, Nixon EE, Fang J, Liu J, Li M, Napadow V, Vangel M, Makris N, et al. Acupuncture mobilizes the brain's default mode and its anti-correlated network in healthy subjects. Brain Res. 2009;1287:84-103.

\section{Ready to submit your research? Choose BMC and benefit from:}

- fast, convenient online submission

- thorough peer review by experienced researchers in your field

- rapid publication on acceptance

- support for research data, including large and complex data types

- gold Open Access which fosters wider collaboration and increased citations

- maximum visibility for your research: over $100 \mathrm{M}$ website views per year

At BMC, research is always in progress.

Learn more biomedcentral.com/submissions 